\title{
Analysis of the Total Heat Exchange with the Aim of Improving the Structure of Cylindrical Boiler Furnaces
}

\author{
K.T. Baubekov \\ S.Seifullin Kazakh agro technical university \\ Astana city, the Republic of Kazakhstan \\ A.K. Baubekova \\ S.Seifullin Kazakh agro technical university \\ Astana city, the Republic of Kazakhstan
}

\begin{abstract}
Annotation - This article gives an analysis of the problems and the prospects for the development of highly efficient and environmentally friendly gas-oil boiler with optimized heat transfer. Given the imperfections of objective indicators used within the combustion methods, which are due to their hyper carcinogenicity and complexity NOx achieve emissions standards in the atmosphere, it offered effective ways to solve the problem by eliminating the hyper trophy heat in the furnace of a conventional boiler. The influence of the rate of heating and rate of cooling gas for the furnace height reveal the essence of the formation of "thermal" NOx. As a result, we found the way of real improvement of new boilers, proposed a simplified method of analysis for the construction of the furnace with the removal of hypertrophied heat so as to avoid the formation of "thermal" NOx reduction and reliability in the most heat-stressed areas of the furnace.
\end{abstract}

Index Terms - hypertrophy of heat exchange, profiling forms of the furnace, preventing the formation of nitrogen oxides.

\section{INTRODUCTION}

Due to the fact that historically the classic configuration of furnaces, types of layouts boilers form convective flues allow to solve the problems associated with the manufacture of boiler heating surfaces technology, their transportation, installation, easy operation and maintenance, maintainability, rational organization of work processes, manufacturers boilers are not interested in achieving an ideal heat transfer processes and improve environmental performance. Not a reasonable change these configurations, layouts and shapes to improve the priority of one of the indicators - reduction of non-uniformity of heat flows - would inevitably lead to the deterioration of the others and will lead to a substantial increase in capital expenditures. In this regard, as shown by calculations require significant work on the final design, the production of new energy boilers and upgrading of new lines in Boiler Plant.

\section{AIMS AND OBJECTIVES}

Our proposed approach for the construction of cylindrical boilers, leveling uneven distribution of heat load on the furnace height, keeping the balance between heat generation and heat removal in some areas of the combustion chamber, allows the calculation of adequate full-scale flare heat receiving surface of the walls of each zone. As a result, we found the way of real improvement of new boilers and recommended a number of simplified designs of environmentally safe boilers, allowing entering the sustainable energy development. From this position, we offer a perfect start small boiler.

\section{DESCRIPTION OF THE RESEARCH SUBJECT}

To analyze and optimize the total heat exchange will take the following assumptions: radiant heat transfer in each section is determined according to the Stefan-Boltzmann equation, and the average temperature in each section is determined from the heat balance of the elementary volume in the medium section. Radiative heat exchange between the individual volumes of the medium, as well as convective heat transfer between the medium and heat perceiving surfaces neglect, because the effect of these factors is not enough. Then the equation of heat balance and heat transfer to the elementary heat perceiving surface can be written as follows (Equation 1) [1] 


$$
\begin{gathered}
q_{\mathrm{v}} \cdot F_{\mathrm{T}} \cdot d h \cdot \varphi= \\
a_{m} \cdot \sigma_{0} \cdot\left(T^{4}-T^{4}{ }_{c m}\right) d H+B_{\mathrm{p}} \cdot v_{\Gamma} \cdot c_{\Gamma} \cdot d T
\end{gathered}
$$

Heat balance equation for the elementary heat perceiving surface can be written as follows (Equation 2)

$$
q_{F} \cdot \pi d_{\mathrm{T}} \cdot d h \cdot \varphi=B_{\mathrm{p}} \cdot v_{\Gamma} \cdot c_{\Gamma} \cdot d T
$$

$q_{\mathrm{v}}$ - heat isolation per unit volume, $\mathrm{kWh} / \mathrm{m}^{3} ; F_{\mathrm{T}}$ - cross-section of the furnace, $\mathrm{m}^{2} ; d h$ - elementary height of the furnace, $\mathrm{m} ; \varphi=1-\frac{q_{5}}{\eta_{\kappa}+q_{5}}$ - heat preservation rate [2]; $a_{m}$ - the apparent emissivity of the furnace; $\sigma_{0}=5,67 \cdot 10^{-11} \mathrm{kWh} /\left(\mathrm{m}^{2} \cdot \mathrm{K}^{4}\right)$ - emissivity blackbody (Stefan-Boltzmann); $T$ - the current absolute temperature of the flame; $T_{c m}$ - the absolute temperature of the beam receiving surface, $\mathrm{K} ; d H$ - the heat receiving surface corresponding to the height, $d h ; B_{p}=\frac{100-q_{4}}{100}$ - estimated fuel combustion, $\mathrm{kg} / \mathrm{s}$ (m3 / s); vg- specific volume of combustion products on $1 \mathrm{~kg}$ of burned fuel, $\mathrm{m}^{3} / \mathrm{kg}$; $\mathrm{C}_{\mathrm{g}}$ - average combustion heat capacity $\mathrm{kJ} /(\mathrm{kg} \cdot \mathrm{K})$; $\mathrm{dT}$ - the increase of temperature in the combustion zone, $\mathrm{K}$.

It is possible to determine the diameter of the furnace (Equation 3)

$$
d_{\mathrm{T}}=\frac{B_{p} \cdot v_{2} \cdot c_{2}}{q_{F} \cdot \pi \cdot \varphi} \cdot \frac{d T}{d h}
$$

$q_{F}$ - surface heat isolation; $d_{\mathrm{T}}$ - the diameter of the combustion chamber.

Replacing the attitude $\frac{d T}{d h}$ of finite differences, we obtain $\frac{\Delta T}{\Delta h}$ (Equation 4)

$$
d_{\mathrm{T}}=\frac{B_{p} \cdot v_{2} \cdot c_{2}}{q_{F} \cdot \pi \cdot \varphi} \cdot \frac{\Delta T}{\Delta h}
$$

According to the heat balance equation of the combustion chamber [2], the amount of heat sensed in the furnace at a fuel $\mathrm{kg} B_{p}$ defined as follows (Equation 5)

$$
\begin{aligned}
& Q_{n}=B_{p} \cdot v_{2} \cdot c_{2} \cdot\left(T_{a}-T_{T}{ }^{n}\right)= \\
& =B_{p} \cdot\left(Q_{T}-I^{\prime \prime}{ }_{T}\right) \cdot \varphi
\end{aligned}
$$

The theoretical combustion temperature, which would take place in an adiabatic (no heat loss to the environment) combustion is defined as follows (Equation 6)

$$
T_{a}=\frac{\pi \cdot d_{\mathrm{T}} \int_{h}^{0} q_{F} \cdot d h}{B_{p} \cdot v_{2} \cdot c_{2}}+T_{\mathrm{T}}{ }^{\prime}
$$

$T_{a}$ - adiabatic combustion temperature, $K ; T_{\mathrm{T}}{ }^{\prime}$ - temperature at the outlet of the furnace, $K$ [2]; $h-$ the height of the furnace zone, defined as the distance from the level of the burner arrangement to the outlet section of the furnace, $\mathrm{m} ; d_{\mathrm{T}}$ - the diameter of the combustion chamber with the theoretical combustion temperature, $\mathrm{m}$.

In order to ensure uniform distribution of the heat load of the heating surfaces, ideally (take for granted) requires changes in the diameter of the zone of the combustion chamber is proportional to the temperature of the flame zone at its height. For a given flame temperature distribution nature of the expression (equation 3 or 4 ) defines the zone of the combustion chamber diameter and make up the profile of a longitudinal section of the combustion chamber. Turn the resulting curve $d_{\mathrm{T}}=\mathrm{f}(\mathrm{h})$ around the vertical axis of the furnace, define the desired shape of the combustion chamber. Thus, the optimum shape of the combustion chamber is a rotation surface of the body, made by the nature of the distribution of the flame temperature at its height and approaches the full-scale form of the torch (Picture 1, a).

Thus, presumably hypertrophy heat can be eliminated in the boiler furnace, taking into account the fact that a legitimate regulatory method $[2,3]$ that the thermal stress on the furnace height area is calculated or 
determined by a factor of uneven distribution of the radiant heat absorption by the furnace height depending on the height h / HT (Picture1, a) and of the average thermal stress of furnace walls of the heating surface (equation 7)

$$
q_{л}^{3}=q_{\pi}^{\mathrm{cp}} \eta_{\beta}, k W / m^{2}
$$

$q_{\pi}{ }^{\mathrm{cp}}$ - the average thermal power of furnace walls of the heating surface.

In accordance with the latest revision of a standard method [3] in case "zoning" or "zoned" firing payment is not made, the specific heat absorption of separate zones can be estimated by the following relationship (8)

$$
q_{n}=y_{6} \frac{B_{p} \cdot Q_{n} \psi}{F_{c m} \psi_{c p}}, \quad \kappa B m / M^{2}
$$

Thus, the proposed scheme of constructing the furnace boiler in order to eliminate the hypertrophy of heat transfer, which consists in profiling furnace boiler adequately full-scale flare, provides in each height of the furnace more uniform (i.e. carrying) the thermal load its radiative heating surfaces [3]. An extension point is no more than $\pm 20 \%$.

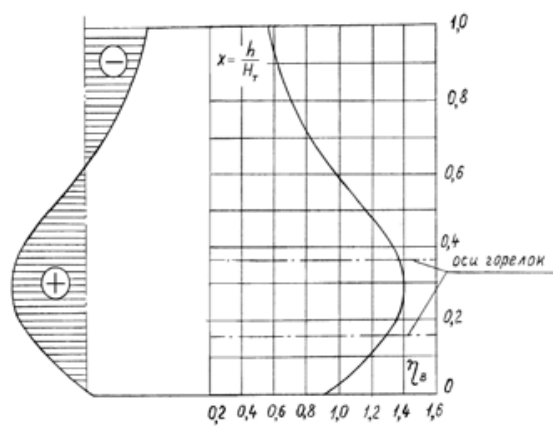

a)

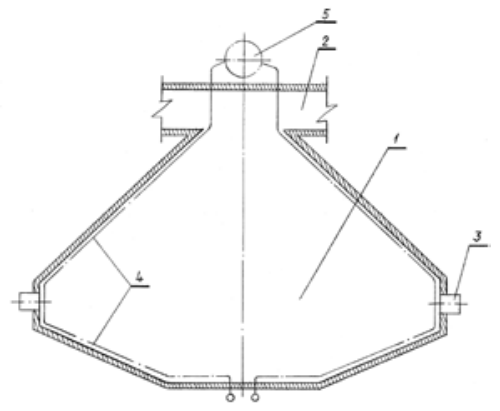

b)

1 - boiler furnace; 2 - convective mine; 3 - burners; 4 radiation heating surface; 5 - boiler drum

Picture 1 - Profiling oil-gas furnace to eliminate hypertrophy: the wall layout burner via heat absorption distribution ratio adjustment furnace [2] (a) and the boiler

TGM-94 alignment unevenness of the specific heat absorption in each area (at a reduced width $b=10,64 \mathrm{M}$ and a constant height of the furnace $H_{m}=20,5 \cdot M$ ) (b).

At the same time, the nature of the family of curves describing the dependence of the main processes that take place in the furnace, and the furnace characteristics identified us with Mathlab program and are subject to the following relationships (equations 9, 10, 11):

- dependence of the distribution of heat on the furnace height $x=\frac{h_{2}}{H_{m}}$ 


$$
\begin{aligned}
& Q_{n}=-76,5 \cdot x^{4}+259 \cdot x^{3}-289 \cdot x^{2}+ \\
& +104 \cdot x+12,6
\end{aligned}
$$

- curve profiling the heat receiving surface of the heating furnace $F$ height to ensure even distribution of the thermal load and prevent the formation of "thermal" nitric oxide

$$
\begin{aligned}
& F=-311 \cdot x^{5}+228 \cdot x^{4}+1153 \cdot x^{3}- \\
& -1787 \cdot x^{2}+697 \cdot x+88
\end{aligned}
$$

- curve effective height of the furnace temperature to ensure even distribution of the thermal load and prevent the formation of "thermal" nitric oxide

$$
\begin{aligned}
& T_{\supset \phi}=1875 \cdot \exp (-0,278 x)- \\
& -371 \cdot \exp (-3,8 x)
\end{aligned}
$$

\section{RESULTS AND PROSPECTS OF FURTHER USE}

The proposed engineering technique zoning alignment thermal stress surface heating furnace walls in width and height of the furnace due to the profiling forms the furnace in proportion to the height of the furnace temperature allows to analyze and achieve the constancy of the thermal stress in each portion of the furnace (see. Table 1), which will lead to the following results: reduction of toxic emissions of nitrogen oxides, soot and carcinogenic polycyclic aromatic hydrocarbons (PAHs); reduce the formation of high-temperature corrosion of metals, screen pipes rupture, reduction in in-line deposits on heat-stressed areas of the most, reduce the overall dimensions and metal boilers. Looking ahead and anticipating the question of structural and technological complexity of the implementation of the proposed boiler, as well as the issue of constructive coupling of the furnace, which is a body of revolution, with the top of the convective flue boiler remember reviewers profiling MEI (Moscow Power Engineering Institute) - boiler furnace CBTI (Central Boiler and Turbine Institute) and RTEI (Russian Thermal Engineering Institute), and that our boilers can also be optimized by a deviation from the ideal form in favor of a priority, but implemented forms (Picture 1, b). For example, to ensure the reliability of the drum of the boiler circulation (E-type) furnace tubes must be lifted up along the walls of the furnace by the shortest route, repeating the firing profile. Moreover, since the furnace has a variable cross-section in the middle of a step between the pipes will be much greater than in the bottom or top of the degree of screening and the coefficient of thermal efficiency of the walls will drop compared to conventional designs. If a step in the middle part between the pipes is uniform, the top of the display screen is necessary in the common collector pipe or perform external cyclone for drum. To find out of this contradictory situation, consider a new model of the furnace (in the case of once-through boilers task easier). Since mainly the task of reducing thermal stress in the active burning zone (ABZ) and a thermal load leveling radiation heating surfaces at the height of the furnace, using the equivalent diameter of each zone (a zone will be enough 3-4) with a constant width parallel furnace can be stepwise or gradually from the bottom to the furnace to lend top (Figure 1). Picture 1b shows the new boiler TGM-94, designed and shaped in this way. 
Table 1 - Estimated alignment of thermal stress of heating the surface of furnace walls with the help of the uneven distribution of the radiant heat absorption coefficient adjustment of the furnace of the boiler TGM-94, depending on the relative height $h$ / $\mathrm{H}_{\mathrm{T}}$

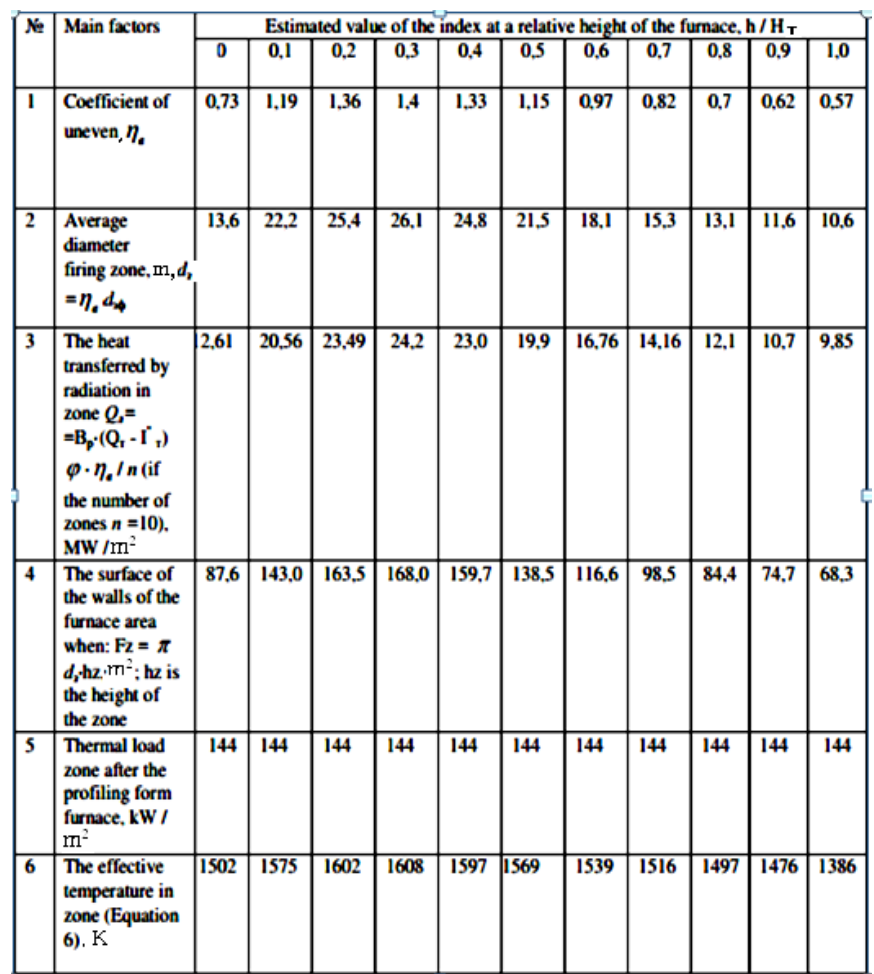

Thus, up to now, we have struggled with the consequence, not the cause of the formation of "thermal" $\mathrm{NO}_{\mathrm{x}}$. We have developed a method of analysis no isothermal inside the furnace processes that reflects the hypertrophy of the total heat transfer in existing boilers, which will prevent the formation of "thermal" $\mathrm{NO}_{\mathrm{x}}$ without increasing the formation of carcinogenic substances by adjusting the rate of heating and cooling gases in some areas of the furnace. Furthermore, studies have shown within combustion $\mathrm{NO}_{\mathrm{x}}$ reduction techniques and evaluation of the sum and each private Ingredients toxicity, reducing toxicity of the combustion products private increases overall toxicity oil-gas boilers [4].

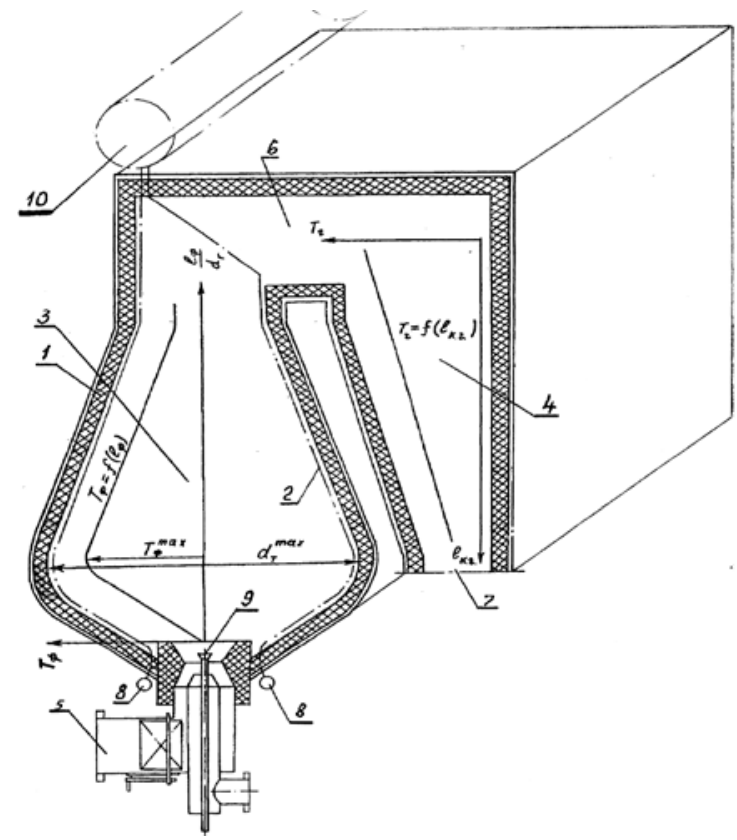

1 - boiler body; 2 - furnace tubes; 3 - combustion chamber; 4 convective flue; 5 adjustable burner; 6- opening; 7 - flue pipe; 8 collectors screen heating surfaces; 9 - oil-fired nozzle; 10- drum 
boiler

Picture 2 - Scheme of construction of the boiler AC equivalent diameter proportional lowering the height temperatures furnace
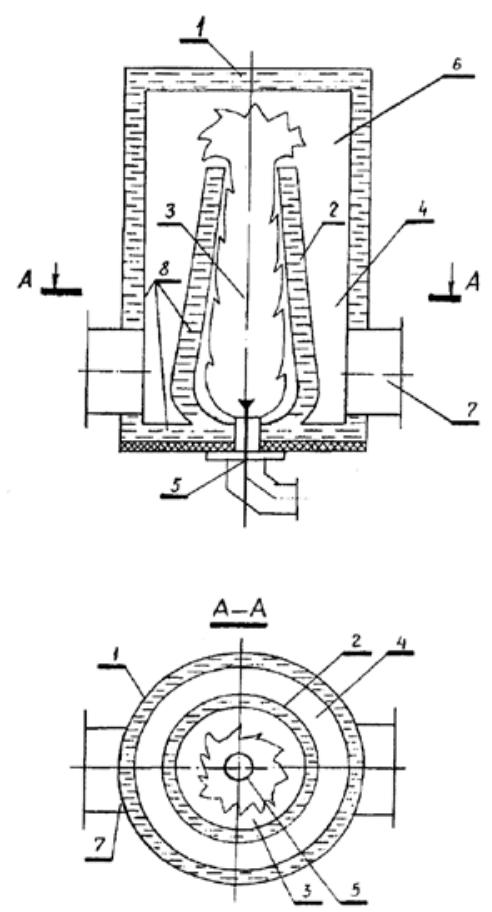

1 - water-cooled housing; 2 - Screen surface made for a given character of zone

flame temperature distribution; 3 - combustion chamber; 4 convective flue (convective bundles conditionally

shown); 5 - burner unit; 6 - opening; 7 - pipe chimney; 8 - water closed space;

Picture 3 - Scheme of construction of the boiler with variable diameter is proportional to the furnace reduction in the height temperature

In this case, the choice of technological measures to suppress the thermal NO in accordance with the MEI and "Heat electrical project" approach [5] will be primarily determined not by the design and layout of the burners of the furnace, and the characteristics of the (ABZ). This means that the suppression of the thermal NO will only be limited by the existing characteristics of the (ABZ), and it is possible that some of the existing boilers will not be achieved even at the level of maximum permissible concentration (MPC) implementing several activities within the flue.

As it is evident from the suggested design of boilers, also increase convective heat transfer can be achieved, for example, changing the shape of the gas flue, reducing the flow area in proportion to the nature of the gas temperature reduction along the length of the flue (picture 2,3 ).

\section{CONCLUSIONS}

Thus, the analysis shows that quite adequate criteria hyper trophy heat playing in combustion processes a large role in the formation of toxicity, safety in the most heat-stressed areas, makes it possible to consider the technological and environmental aspects of this issue and improve the boiler design through the design analysis and refinement hyper trophy criterion heat.

\section{REFERENCES}

[1] Bloch A.G., Zhuravlev Yu.A., Ryzhkov L.N. Radiation heat transfer: A Handbook. M.: Energoatomisdat, 1991. - 432 p.

[2] Thermal design of boiler units (standard method). 2nd ed. reprod.- M .: Energia, 1973. - 296 p.

[3] Thermal design of boiler units (standard method). 3rd ed. Revised. and ext. SPb .: CKTI NGOs, 1998. - 257 p.

[4] Baubekov K.T. Environmental safety and indicators of toxicity oil-gas boilers // Ecological safety of Russia's regions and the risk of 
International Journal of Innovations in Engineering and Technology (IJIET) http://dx.doi.org/10.21172/ijiet.82.032

man-caused accidents and disasters: a collection of articles X International scientific-practical conference. - Penza: Volga house Knowledge, 2010. - P. 80-86.

[5] Roslyakov P.V., Dvoinishnikov V.A., Zelinsky A.E., Timofeev S., Burkov V.Y., Nazdryuhina G.V. / Development of recommendations to reduce NOx emissions for gas-oil thermal power plant boilers // Electric stations. - Moscow, 1991. - № 9. - S. 917.

[6] Davidzon M.I. The effect of heat flow density on the formation of in-fat // Thermal Engineering. - Moscow, 2001. - № 1. - S. 72-73. 